Rev. Téc. Ing. Univ. Zulia. Vol. 44, No. 2, Mayo-Agosto, 2021, 75-82

\title{
Eficiencia de Uso del Agua en Riego por Goteo Superficial y Subsuperficial en Zea mays L.
}

\section{José Lauro Conde-Solano ${ }^{1}$ (D), Adriana Beatriz Sánchez-Urdaneta ${ }^{2}$ (D), Ciolys Beatriz Colmenares de Ortega ${ }^{2 *}$ (D) , Jorge Ortega-Alcalá ${ }^{(\mathbb{D})}$ y Edison Ramiro Vásquez ${ }^{3}$ (D)}

${ }^{1}$ Facultad de Ciencias Agropecuarias, Universidad Técnica de Machala. Machala, Ecuador.

${ }^{2}$ Departamentos de Botánica y Estadística, Facultad de Agronomía, Universidad del Zulia. Maracaibo, Venezuela.

${ }^{3}$ Universidad Nacional de Loja. Loja, Ecuador.

*Autor de correspondencia: ciolysc@gmail.com https://doi.org/10.22209/rt.v44n2a02

Recepción: 08 de septiembre de 2020 | Aceptación: 12 de febrero de 2021| Publicación: 01 de abril de 2021

\section{Resumen}

En la producción de alimentos el agua constituye un factor limitante; por ello, es preciso alcanzar la máxima eficiencia del uso de este recurso en los sistemas agrícolas. Se evaluó la eficiencia del uso de agua del riego por goteo superficial y subsuperficial en el cultivo de Zea mays L. Se cultivaron $1.600 \mathrm{~m}^{2}$ de maíz híbrido duro, con cuatro tratamientos: riego por goteo superficial (T1, testigo) y subsuperficial a 10 (T2), 20 (T3) y 30 (T4) cm de profundidad, con cuatro repeticiones. En un diseño en bloques completamente al azar, cada unidad experimental tuvo $100 \mathrm{~m}^{2}$ (10 m x $\left.10 \mathrm{~m}\right)$. Se realizó un análisis de varianza y comparación de medias mediante la prueba de Tukey. Se evaluó el rendimiento, eficiencia del uso del agua (EUA), frecuencia, tiempo y lámina de riego, y diámetro del bulbo humedecido. Hubo diferencias estadísticas para todas las variables evaluadas $(\mathrm{p}<0,01)$, excepto para la lámina de agua por riego por efecto de los tratamientos. El rendimiento $(10.263 \mathrm{~kg} / \mathrm{ha})$, EUA $\left(7,92 \mathrm{~kg} / \mathrm{m}^{3}\right)$ y diámetro $(0,145 \mathrm{~m})$ del bulbo húmedo fueron mayores en T3 y T4; la frecuencia de riego (3,6 días) y la lámina total de agua fue menor en T3 y T4 (129 mm); el tiempo total de riego fue menor en T4 (34,08 h). El riego por goteo subsuperficial a 20 y $30 \mathrm{~cm}$ de profundidad presentó la mayor eficiencia.

Palabras clave: escasez de agua; maíz, sistemas agrícolas; rendimiento; tiempo de riego.

\section{Water use Efficiency in Surface and Subsurface Drip Irrigation in Zea mays $\mathrm{L}$.}

\begin{abstract}
In food production, water is a limiting factor; therefore, it is necessary to achieve maximum efficiency in the use of this resource in agricultural, systems. The efficiency of the water use of surface and subsurface drip irrigation in the Zea mays L. crop was evaluated. 1,600 $\mathrm{m}^{2}$ of hard hybrid corn were cultivated, with four treatments: superficial drip irrigation (T1, control) and subsurface at 10 (T2), 20 (T3) and 30 (T4) cm deep and four repetitions. In a randomized block design, each experimental unit was $100 \mathrm{~m}^{2}(10 \mathrm{~m}$ x $10 \mathrm{~m})$. The yield, water use efficiency (WUE), frequency, time and irrigation sheet, diameter of the wetted bulb were measured. There were statistical differences for all the variables evaluated ( $\mathrm{p}<0.01$ ), except for the sheet of water per irrigation due to the effect of the treatments. Yield $(10,263 \mathrm{~kg} / \mathrm{ha}), \mathrm{WUE}\left(7.92 \mathrm{~kg} / \mathrm{m}^{3}\right)$, diameter wet bulb $(0.145 \mathrm{~m})$ were higher in T3 and T4; the frequency of irrigation (3.6 days) and the total sheet of water was lower in T3 and T4 (129 mm); the total irrigation time was shorter in T4 (34.08 h). Subsurface drip irrigation at 20 and $30 \mathrm{~cm}$ depth showed the highest efficiency.
\end{abstract}

Keywords: agricultural systems; maize; water scarcity; yield; irrigation time. 


\section{Introducción}

La demanda mundial de agua se ha incrementado anualmente $1 \%$ con relación al acrecentamiento de la población, el desarrollo económico y los cambios en los patrones de consumo, entre otros factores (WWAP, 2018). De acuerdo con de Miguel y Tavares (2015), el desafío ambiental acerca de la gestión del agua, requiere alcanzar un equilibrio, priorizando entre el crecimiento económico, la disminución de la pobreza, la conservación del agua y el cambio climático; no obstante, se sigue manejando independientemente de la gestión de los ecosistemas; por lo cual, se requiere un abordaje integral, destacando la importancia del agua en los ecosistemas y la relación de su gestión y manejo, con una perspectiva integral.

La escasez del recurso hídrico en muchas regiones del mundo, especialmente en las zonas áridas y semiáridas, el alto costo de utilización y la demanda de productos alimenticios agrícolas, requeridos por una creciente población, hace que se busquen alternativas para mejorar la eficiencia de uso o productividad del agua, especialmente en la agricultura, debido a que este sector consume grandes volúmenes de este líquido, con el agravante de que un alto porcentaje de este recurso representa uso consuntivo para la planta (escorrentía, percolación profunda, evaporación directa del suelo y evapotranspiración, entre otros) (Gomes et al., 2011; Nieto et al., 2018; Papanatsiou et al., 2019). Por lo que, Sánchez y Rivera (2018) indicaron que se hace necesario utilizar y poner en práctica nuevas formas de aplicación en el riego, que se conocen son eficientes, con lo cual se optimice el recurso hídrico y se obtenga una mayor productividad y rendimiento de los cultivos.

Según la Organización de las Naciones Unidas para la Alimentación y la Agricultura (FAO, 2019), la causa de la escasez de este preciado líquido está dada principalmente por la agricultura; esencial para la seguridad alimentaria, lo cual representa el $70 \%$ de la extracción de este recurso, alcanzando hasta $95 \%$ en algunos países en vías de desarrollo. El incremento de su escasez representa un desafío para el desarrollo sostenible. Los recursos de agua dulce disminuyen de manera alarmante, esto sugiere que dos tercios de la población mundial para el 2025 podrían estar viviendo en países con estrés hídrico, de continuar los patrones de consumo actuales.

En Ecuador, y en particular en la región sur del país, existen zonas donde las precipitaciones oscilan entre 200 y $600 \mathrm{~mm} / \mathrm{año} \mathrm{(Plan} \mathrm{de} \mathrm{Desarrollo} \mathrm{y} \mathrm{Ordenamiento} \mathrm{Territorial} \mathrm{de} \mathrm{la} \mathrm{Provincia} \mathrm{de} \mathrm{El} \mathrm{Oro,} \mathrm{2015),} \mathrm{siendo} \mathrm{evidente} \mathrm{la} \mathrm{escasez} \mathrm{de}$ agua; convirtiéndose su disponibilidad en la principal limitante para la producción agrícola.

La evaluación de las diferentes alternativas o métodos de riego, es necesaria para lograr una máxima eficiencia en el uso del agua. Todas las alternativas de riego clásicas aplican grandes cantidades de agua para suplir las necesidades de esta en los cultivos, debido a su alto consumo, especialmente por evaporación. El riego por goteo subsuperficial puede evitar el consumo excesivo de agua al reducir las pérdidas de aplicación (evaporación) (Ayars et al., 2015; Eranki et al., 2017; Sinha et al., 2017; Bringas-Burgos et al., 2020), ya que es suministrado a la profundidad donde se desarrolla el sistema radical del cultivo, formándose el bulbo húmedo en la parte subsuperficial del suelo, evitando estar en contacto directo con la radiación solar (Lucero-Vega et al., 2017). Al-Ghobari y Dewidar (2018) indicaron que con este sistema de riego fueron reducidos entre 20 y 40 \% los requerimientos hídricos de las plantas, y las aplicaciones de agua fueron uniformes.

En el riego por goteo subsuperficial la principal ventaja es que se conserva el contenido de humedad en el suelo, utilizando bajos volúmenes con altas frecuencias, con lo que se permite el óptimo crecimiento de las plantas, aspecto relevante porque la mayor limitante para la producción agrícola en zonas semiáridas y áridas es la disponibilidad de agua (Montemayor et al., 2006).

El maíz como grano y forraje constituye uno de los cereales más importantes para el consumo humano, animal, en la farmacéutica y en la producción industrial. En Ecuador, el maíz corresponde a uno de los principales cultivos de ciclo corto, se desarrolla en diferentes pisos altitudinales, por lo que se adapta a diversos ambientes. El Instituto Nacional de Estadística y Censos (INEC, 2019), señaló que la superficie sembrada de maíz duro seco a nivel nacional en el 2018 fue de 383.399 ha; la producción se concentró en la provincia de los Ríos $(45,4$ \%) con 602.000 t.

Debido a que el maíz es un cultivo C4, su eficiencia en el uso del agua es elevada; esto producto de la alta tasa de fotosíntesis, con un aporte mínimo de la tasa de transpiración ligeramente restringida (Steduto et al., 2007). También destaca que bajo condiciones de riego y alta fertilidad alcanzó rendimientos entre 11.000 y 14.000 kg/ha (Hsiao y Fereres, 2012).

Es claro que el agua es un factor de desarrollo productivo, en donde deberían confluir los intereses de los expertos en planificación y manejo de los recursos hídricos con fines de riego, usuarios del agua para riego, gobernantes, administradores del agua, sociedad en general, y encontrar los medios para que este recurso genere el mayor beneficio económico y social. 
El uso del agua en la agricultura se debe realizar de manera eficiente, productiva, equitativa y respetuosa del ambiente. Se trata de producir más alimentos utilizando menos agua, aumentar la resiliencia de las comunidades agrícolas para hacer frente a las inundaciones y las sequías, y la aplicación de tecnologías limpias que protejan el ambiente. El problema de la escasez de agua es un aspecto fundamental del desarrollo sostenible.

Howell (2001) señaló que las pérdidas por evaporación, escurrimiento y percolación fueron minimizadas a través de la microirrigación; además, de hacer más eficiente el riego realizando aplicaciones cortas y frecuentes; aprovechando la fertirrigación para proveer los nutrientes que el cultivo requiere y aumentar la producción.

Con base en estos fundamentos anteriores, el presente trabajo tuvo como objetivo evaluar la eficiencia del uso del agua aplicada a través de riego por goteo superficial y subsuperficial en el cultivo de maíz híbrido duro.

\section{Materiales y Métodos}

El ensayo se desarrolló en el área experimental de la hacienda Santa Inés, Facultad de Ciencias Agropecuarias, Universidad Técnica de Machala, ubicada en el km 5 1/2 vía Pasaje, provincia de El Oro, zona de planificación 7, Ecuador; entre las coordenadas 6200000 y 9638000 S y 6202000 y 9637800 S, zona geográfica 17 S, proyección universal transversa de Mercator, donde termina la planicie aluvial de la cuenca del río Jubones.

El clima es tropical megatérmico semihúmedo, con una altitud de $5 \mathrm{msnm}$, temperatura media de $25^{\circ} \mathrm{C}$, mientras que la precipitación media registra $600 \mathrm{~mm}$ con dos períodos pluviométricos bien marcados, el lluvioso que inicia generalmente en enero y termina en abril y el seco que va de mayo a diciembre. La evapotranspiración de referencia en esta zona es de 1300 a $1500 \mathrm{~mm}$, el déficit hídrico anual oscila entre 225 y 925 mm (Plan de Desarrollo y Ordenamiento Territorial de la Provincia de El Oro, 2015). El suelo es franco-limoso en los primeros $30 \mathrm{~cm}$ de profundidad.

El material vegetal utilizado fue el maíz híbrido duro (híbrido PIONEER@ 30K75), sembrado a una distancia de $80 \mathrm{~cm}$ entre surcos y $40 \mathrm{~cm}$ entre plantas, con dos semillas por punto. En cuanto a las características vegetativas de la clase híbrido simple, presenta hojas semi-erectas, alcanza una altura media de 2,50 m, periodo vegetativo semi-tardío de $125-135$ días, inserción de la mazorca a 1,30 m, relación grano/mazorca desgranada 85/15.

El diseño del experimento fue de bloques completos al azar, con cuatro tratamientos y cuatro repeticiones. El experimento se estableció en una superficie total cultivada de maíz de $1.600 \mathrm{~m}^{2}$, conteniendo 16 unidades experimentales de $100 \mathrm{~m}^{2}$ (10 m de largo x $10 \mathrm{~m}$ de ancho). Los tratamientos utilizados fueron: riego por goteo superficial (T1, testigo), riego por goteo subsuperficial a 10 (T2), 20 (T3) y a 30 (T4) cm de profundidad. Las zanjas para la instalación del sistema de riego se realizaron en forma manual.

La programación del riego se realizó con la finalidad de responder adecuadamente cuándo regar y qué cantidad de agua aportar. El sistema de riego fue independiente para cada tratamiento, controlado por una válvula de compuerta. La lámina suministrada fue registrada a través de válvulas volumétricas de precisión, las frecuencias y tiempos de riego se realizaron en función de las lecturas de los tensiómetros instalados para cada tratamiento, los tensiómetros fueron calibrados antes de ser instalados.

El caudal nominal de los goteros fue de 1,65 l/h, con una variación $\pm 5 \%$, dado por la casa fabricante (Hydrodrip Super Flat Integral Dripline, PLASTRO), cuya presión de trabajo fue de $10 \mathrm{mca}$. Fueron instalados a $80 \mathrm{~cm}$ entre laterales de riego y $50 \mathrm{~cm}$ entre goteros, para humedecer una franja horizontal continua. Los laterales de riego fueron de polietileno de $16 \mathrm{~mm}$ de diámetro, la tubería secundaria de polietileno de $32 \mathrm{~mm}$ de diámetro, la tubería principal de PVC de $40 \mathrm{~mm}$ de diámetro, la energía o presión suministrada al sistema fue de 12 mca a la salida del cabezal de riego a través de un equipo motobomba, abastecido por un pozo subterráneo.

Se determinó el rendimiento del cultivo al registrar la biomasa del grano seco de 40 plantas por tratamiento, con un total de 160 plantas por tratamiento; la eficiencia de uso del agua para riego o también denominada productividad del agua, fue definida en función del rendimiento en $\mathrm{kg}$ de producto de maíz en grano seco por $\mathrm{m}^{3}$ de agua utilizada. Se utilizó la siguiente ecuación:

$$
\text { Eficiencia de uso del agua }=\frac{\text { Producción }(\mathrm{kg})}{\text { Agua utilizada }\left(\mathrm{m}^{3}\right)}
$$

Para determinar el contenido de humedad óptimo para la planta, se instalaron 16 tensiómetros (Irrometer@) en cada uno de los bloques y tratamientos, calibrados previamente en el sitio del ensayo. Para este proceso se instaló el tensiómetro en suelo cuyo contenido de humedad estuvo a capacidad de campo, para cada lectura del tensiómetro se 
determinó el contenido de humedad del suelo con base a su peso a través del método gravimétrico (Figura 1). Cuando la curva se tornaba horizontal, la lectura del tensiómetro (cbar) indicó que la planta necesitaba riego; principio que se utilizó para su aplicación, cuyas lecturas indicaron el potencial matricial del suelo o la tensión con la que el suelo retuvo las partículas de agua; este dispositivo, según Ferreyra et al. (2006) y Girona et al. (2006), permitió controlar la magnitud del estrés hídrico, ya sea por exceso o déficit de agua.

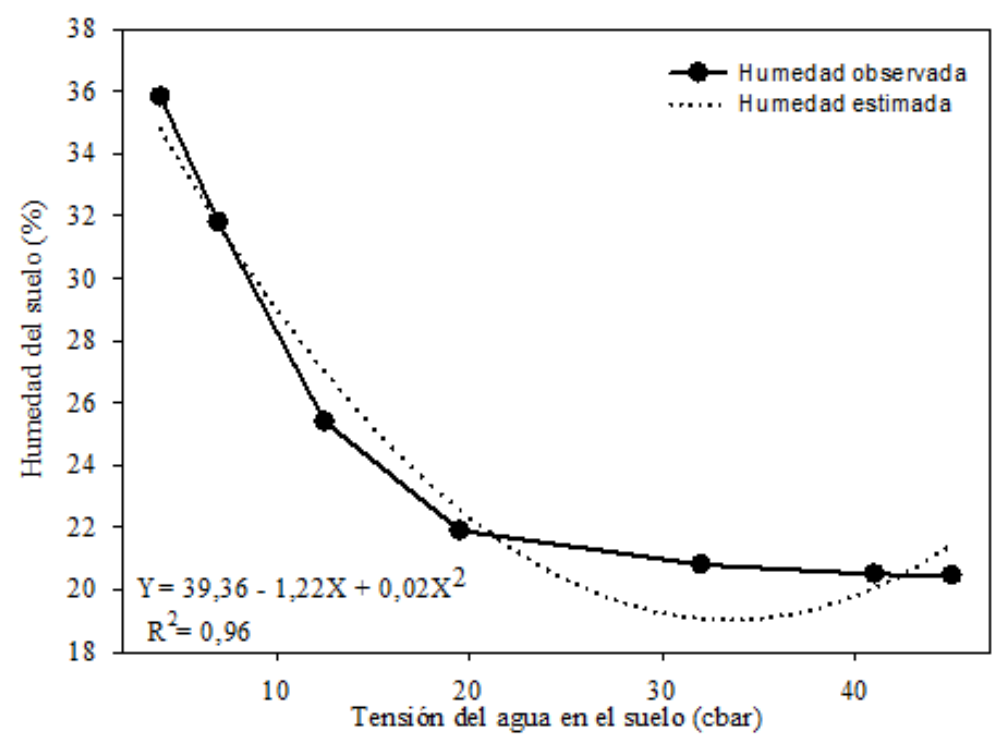

Figura 1. Curva de retención de humedad del suelo, granja experimental UTMACH.

Los tensiómetros se instalaron a $20 \mathrm{~cm}$ de profundidad dentro del bulbo húmedo del gotero debido a que a esa profundidad se encuentra el mayor porcentaje de raíces del sistema radical. El riego fue suministrado cuando la lectura del manómetro indicó 45 cbar, y cuando este marcó 10 cbar el suministro de riego se interrumpió, lo que indicó que el contenido de humedad estaba a capacidad de campo.

Para determinar el diámetro del bulbo húmedo de los emisores instalados en forma superficial y subsuperficial (10, 20 y $30 \mathrm{~cm}$ de profundidad), se excavó, y para medir se utilizó un flexómetro. Las medidas se tomaron a partir del eje central, partiendo de este, se midió en diferentes profundidades hacia los extremos, y se obtuvieron las medias de los diferentes diámetros humedecidos obtenidos, proceso que se realizó una vez terminado el ciclo del cultivo, con el fin de no interferir en el desarrollo radical y foliar normal de la planta.

A cada variable se le realizó un análisis de varianza mediante la prueba de F, para identificar diferencias estadísticamente significativas en los efectos de los tratamientos. Para determinar el mejor tratamiento (o los mejores), se compararon las medias de los tratamientos mediante la prueba de rango múltiple de medias de Tukey $(p \leq 0,05)$. Los análisis estadísticos se realizaron utilizando el paquete estadístico SAS®, versión 15.1 (Statistical Analysis System, 2020).

\section{Resultados y Discusión}

Se encontraron diferencias estadísticas significativas en los efectos de los tratamientos para la variable rendimiento $(\mathrm{kg} / \mathrm{ha} ; p<0,024)$ y para la eficiencia en el uso del agua $(\mathrm{kg} / \mathrm{m} 3 ; p<0,002)$. Con respecto a la respuesta en el rendimiento del cultivo al efecto de los tratamientos, se generaron dos grupos de tratamientos sin diferencias estadísticas significativas al interior de cada grupo $(p>0,05)$, pero con diferencias estadísticamente significativas entre los grupos $(p<0,01)$. Uno de los grupos estuvo conformado por los tratamientos de riego por goteo subsuperficial a los 20 y $30 \mathrm{~cm}$ de profundidad, el otro grupo se constituyó con los tratamientos de sistemas de riego por goteo superficial y subsuperficial a $10 \mathrm{~cm}$ de profundidad (Tabla 1). 
Tabla 1. Rendimiento en grano seco del maíz (Z. mays L.) en $\mathrm{kg} / \mathrm{ha} \mathrm{y} \mathrm{uso} \mathrm{eficiente} \mathrm{del} \mathrm{agua} \mathrm{en} \mathrm{kg} / \mathrm{m}^{3}$ por efecto de la aplicación de riego superficial y subsuperficial a 10, 20 y $30 \mathrm{~cm}$ de profundidad.

\begin{tabular}{ccc}
\hline Tratamientos & Rendimiento (kg/ha) & $\begin{array}{c}\text { Eficiencia de uso del } \\
\text { agua }\left(\mathbf{k g} / \mathbf{m}^{3}\right)\end{array}$ \\
\hline $\begin{array}{c}\text { Riego por goteo subsuperficial a } 20 \mathrm{~cm} \text { de } \\
\text { profundidad (T3) }\end{array}$ & $10.337,5 \mathrm{a}$ & $7,95 \mathrm{a}$ \\
$\begin{array}{c}\text { Riego por goteo subsuperficial a } 30 \mathrm{~cm} \text { de } \\
\text { profundidad (T4) }\end{array}$ & $10.189,1 \mathrm{a}$ & $7,89 \mathrm{a}$ \\
$\begin{array}{c}\text { Riego por goteo superficial (T1) } \\
\text { Riego por goteo subsuperficial a } 10 \mathrm{~cm} \text { de } \\
\text { profundidad (T2) }\end{array}$ & $9.259,4 \mathrm{~b}$ & $6,10 \mathrm{~b}$ \\
\hline
\end{tabular}

Medias con letras iguales en la misma columna, no presentan diferencias estadísticas $(p \leq 0,05)$, según la prueba de Tukey por efecto de los tratamientos aplicados.

El mayor rendimiento lo presentaron los tratamientos de riego por goteo subsuperficial a 20 y $30 \mathrm{~cm}$ de profundidad, con una media entre ambos de $10.263,3 \mathrm{~kg} / \mathrm{ha}$, seguido de los tratamientos por goteo superficial y subsuperficial a 10 $\mathrm{cm}$ de profundidad $(9.246,1 \mathrm{~kg} / \mathrm{ha})$, siendo superior el primer grupo con un poco más de $1.000 \mathrm{~kg}$ de diferencia; esto es, 1,11 veces mayor en el grupo 1 con respecto al grupo 2 (Tabla 1). Con respecto a esta variable, se cotejó el rendimiento experimental de los mejores tratamientos con los rendimientos regionales, tomando como referencia los rendimientos oficiales reportados para la provincia de El Oro en 2019, que fueron de 5.207,57 kg/ha (INEC, 2020). Los rendimientos estimados en esta investigación correspondieron casi al doble, de los obtenidos para la media regional. Esto equivalió a 1,77 y 1,99 veces mayor para los tratamientos de riego por goteo subsuperficial a 10 y $20 \mathrm{~cm}$ de profundidad, respectivamente.

De acuerdo con Zamora-Salgado et al. (2011), se ha considerado que bajo condiciones de riego un rendimiento medio entre 6 y 9 t/ha, podría ser aceptable como producción comercial, con un contenido de humedad del grano entre 10 a $13 \%$, lo cual coincidió con los resultados obtenidos en esta investigación, donde el contenido de humedad del grano fue de $13 \%$.

La eficiencia de uso del agua o productividad del agua suministrada a través del riego por goteo superficial y subsuperficial $(10,20$ y $30 \mathrm{~cm}$ de profundidad), fueron de 6,10;6,63; 7,95 y 7,89 kg/m³; respectivamente (Tabla 1). Los tratamientos de riego por goteo subsuperficial a 20 y $30 \mathrm{~cm}$ de profundidad no presentaron diferencias estadísticas entre ellos $(p>0,05)$, pero fueron diferentes al compararlos con el riego por goteo a $10 \mathrm{~cm}$ de profundidad y el superficial, sin diferencias entre estos dos últimos ( $p>0,05$; Tabla 1$)$. No obstante, el riego por goteo subsuperficial a $20 \mathrm{~cm}$ de profundidad, mostró los mayores valores de eficiencia en el uso del agua y rendimiento en el cultivo de maíz.

En esta investigación el rendimiento en el riego subsuperficial a $20 \mathrm{~cm}$ de profundidad fue 1,43 veces mayor que el riego subsuperficial a $30 \mathrm{~cm}$ de profundidad; 10,43\% mayor que el riego por goteo superficial y 10,69 \% con respecto al riego subsuperficial a $10 \mathrm{~cm}$ de profundidad, que fue el que presentó la menor producción. Igualmente, la productividad máxima del agua fue de 7,95 kg/m $\mathrm{m}^{3}$ en el riego subsuperficial a $20 \mathrm{~cm}$ de profundidad, lo cual correspondió a 0,75; 16,60 y $23,27 \%$ de un mayor ahorro de agua al compararlo con riego por goteo subsuperficial a 30 y $10 \mathrm{~cm}$ de profundidad, y riego por goteo superficial.

Zamora et al. (2007) y Zamora-Salgado et al. (2011) reportaron una media de eficiencia del uso del agua aplicada por goteo en el cultivo de maíz híbrido de $2,53 \mathrm{~kg} / \mathrm{m}^{3}$, mientras que Salomó et al. (2019) obtuvieron una eficiencia media de uso del agua en tres años de evaluación de 2,81 y $2,5 \mathrm{de} \mathrm{kg} / \mathrm{m}^{3}$ para goteo subsuperficial a 25 y $35 \mathrm{~cm}$ de profundidad; respectivamente, en tanto que para riego por inundación fue de $2,45 \mathrm{~kg} / \mathrm{m}^{3}$; contrastando ampliamente con los resultados obtenidos en esta investigación, donde los valores obtenidos fueron superiores 2,76 veces.

No hubo diferencias estadísticas significativas $(p>0,05)$ para la lámina de agua aplicada por riego (mm) por efecto de los tratamientos, mientras que para las variables frecuencia de riego (días), tiempo medio por riego (horas), tiempo total de riego (horas), lámina de agua total aplicado $(\mathrm{mm})$ y diámetro del bulbo humedecido $(\mathrm{m})$, se presentaron diferencias significativas por efecto de los tratamientos $(p<0,05)$. La frecuencia de riego conformó dos grupos, uno constituido por el riego por goteo subsuperficial a 20 y $30 \mathrm{~cm}$ de profundidad, sin diferencias entre ellos, pero diferentes estadísticamente al riego por goteo superficial y subsuperficial a $10 \mathrm{~cm}$ de profundidad. Allí se destacó que la frecuencia de riego fue entre 3,0 días (riego por goteo superficial y subsuperficial a $10 \mathrm{~cm}$ de profundidad) y 3,6 días (riego por goteo a 20 y $30 \mathrm{~cm}$ de profundidad, Tabla 2). 
Tabla 2. Frecuencia, tiempo, lámina de riego y diámetro del bulbo humedecido por efecto de la aplicación de riego superficial y subsuperficial a 10, 20 y $30 \mathrm{~cm}$ de profundidad.

\begin{tabular}{|c|c|c|c|c|}
\hline \multirow{3}{*}{ Profundidad } & \multicolumn{4}{|c|}{ Riego por goteo } \\
\hline & \multirow{2}{*}{$\begin{array}{c}\text { Superficial } \\
\mathbf{0 ~ c m}\end{array}$} & \multicolumn{3}{|c|}{ Subsuperficial } \\
\hline & & $10 \mathrm{~cm}$ & $20 \mathrm{~cm}$ & $30 \mathrm{~cm}$ \\
\hline Frecuencia de riego (días) & $3,00^{b}$ & $3,00^{b}$ & $3,60^{\mathrm{a}}$ & $3,60^{\mathrm{a}}$ \\
\hline Tiempo medio por riego (horas) & $1,20^{\mathrm{a}}$ & $1,11^{\mathrm{b}}$ & $1,24^{\mathrm{a}}$ & $1,21^{\mathrm{a}}$ \\
\hline Tiempo total de riego (horas) & $40,20^{\mathrm{a}}$ & $36,48^{\mathrm{ab}}$ & $34,78^{\mathrm{ab}}$ & $34,08^{b}$ \\
\hline $\begin{array}{l}\text { Lámina de agua aplicada por riego } \\
\qquad(\mathrm{mm})\end{array}$ & $4,6^{\mathrm{a}}$ & $4,2^{\mathrm{a}}$ & $4,7^{\mathrm{a}}$ & $4,6^{\mathrm{a}}$ \\
\hline Lámina de agua total aplicada (mm) & $152^{\mathrm{a}}$ & $139^{\mathrm{ab}}$ & $130^{\mathrm{b}}$ & $129^{\mathrm{b}}$ \\
\hline Diámetro del bulbo humedecido (m) & $0,412^{\mathrm{b}}$ & $0,420^{\mathrm{b}}$ & $0,442^{\mathrm{a}}$ & $0,447^{a}$ \\
\hline
\end{tabular}

Las letras diferentes dentro de cada fila indican que hubo diferencias estadísticas, de acuerdo a la prueba múltiple de medias de Tukey $(p<0,05)$ por efecto de los tratamientos aplicados.

El agua aplicada se encuentra relacionada con la etapa fenológica y la respuesta fisiológica del cultivo a la producción y calidad de la cosecha obtenida. Esto implica, menor cantidad de agua en algunos periodos de desarrollo de la planta, lo cual permite predecir cuándo y cuánto regar. En general, las plantas responden al potencial de agua en el suelo y no directamente a la frecuencia de riego, lo que hace cada vez más importante la determinación del contenido del agua en el suelo, el estado hídrico de la planta y el balance hídrico del continuo suelo-planta-atmósfera.

El tiempo medio por riego fue entre 1,1 y $1,24 \mathrm{~h}$; donde el riego por goteo subsuperficial a 20 y $30 \mathrm{~cm}$ de profundidad y el riego por goteo superficial, no presentaron diferencias significativas $(p>0,05)$ entre ellos, pero al compararlos con el riego por goteo subsuperficial a $10 \mathrm{~cm}$ de profundidad, fueron estadísticamente diferentes $(p<0,01)$. En este sentido, el tiempo de riego en el riego por goteo subsuperficial a $20 \mathrm{~cm}$ de profundidad fue 2,42; 3,23 y 10,48 \% mayor que el riego por goteos subsuperficial a $30 \mathrm{~cm}$ de profundidad, el riego por goteo superficial y el riego por goteo subsuperficial a $10 \mathrm{~cm}$ de profundidad, respectivamente (Tabla 2).

Hubo diferencias estadísticas significativas $(p<0,01)$ para el tiempo total de riego entre el riego por goteo subsuperficial a $30 \mathrm{~cm}$ de profundidad y el riego por goteo superficial, mientras que el riego por goteo subsuperficial a 10 y $20 \mathrm{~cm}$ de profundidad, fueron similares entre sí y sin diferencias estadísticas entre los dos tratamientos anteriores, el tiempo total de riego estuvo entre 40,2 y 34,08 h (Tabla 2). Destaca que el riego por goteo superficial requirió 9,25; 13,48 y 15,22 \% más tiempo de riego, lo cual representó 3,72; 5,42 y 6,12 h con respecto al riego por goteo subsuperficial a 10, 20 y $30 \mathrm{~cm}$ de profundidad en ese orden. Esto sugiere un menor tiempo de uso de los equipos de bombeo, de consumo de agua y electricidad, para cumplir con esta labor en el cultivo al utilizar riego subsuperficial.

La lámina de agua aplicada por riego estuvo entre 4,2 y 4,7 mm. No obstante, las láminas totales aplicadas al cultivo de maíz hasta los 100 días después de la siembra a través del riego por goteo superficial y subsuperficial a 10, 20, y $30 \mathrm{~cm}$ de profundidad, fueron de 152, 139, 130 y $129 \mathrm{~mm}$, correspondientemente (Tabla 2), lo cual correspondió a una diferencia de 8,55; 14,47 y 15,13 \% al compararlo con el riego por goteo superficial, que presentó la mayor lámina de agua aplicada con el riego por goteo subsuperficial a 10, 20 y $30 \mathrm{~cm}$ de profundidad, respectivamente. Esto representó una diferencia de 13, 22 y $23 \mathrm{~mm}$ de agua al mantener la misma comparación entre el riego por goteo superficial, con respecto al riego por goteo subsuperficial a 10, 20 y $30 \mathrm{~cm}$ de profundidad, en el orden dado.

Se ha señalado que, al comparar el riego por goteo subterráneo con el riego tradicional (gravedad), el agua de riego destinado para el cultivo del maíz se redujo de 35 a 55 \% (Lamm y Trooienm, 2003), mientras que Montemayor et al. (2007) indicaron que en maíz forrajero, al comparar el riego por goteo sub-superficial con el riego superficial, hubo un ahorro de agua de 27,4 \%; coincidiendo estos autores que en el riego por gravedad se presentó una mayor pérdida de agua.

También destaca que con la implementación del sistema de riego superficial y subsuperficial a $10 \mathrm{~cm}$ de profundidad, se mantuvo la superficie del suelo relativamente seca, mientras que a 20 y $30 \mathrm{~cm}$ de profundidad, se observó totalmente seca. Al respecto, Thompson et al. (2009) indicaron que hubo un control significativo de los arvenses, se disminuyó la lixiviación de $\mathrm{NO}_{3}$ - y los rendimientos fueron mayores al compararlos con el riego superficial; atribuyéndolo a que el agua y los nutrientes alcanzaron la parte más activa de las raíces. 
Con respecto al diámetro del bulbo humedecido, este varió entre 0,412 y 0,447 m; en esta variable los valores indicados correspondieron al riego por goteo superficial y el riego por goteo subsuperficial a $30 \mathrm{~cm}$ de profundidad, respectivamente. Sobre la superficie del suelo donde estaban ubicados los tratamientos de riego por goteo superficial y subsuperficial a $10 \mathrm{~cm}$ de profundidad, se observó el humedecimiento del bulbo, mientras que con el riego subsuperficial a mayor profundidad (20 y $30 \mathrm{~cm}$ ), esto no fue posible, lo que guardó relación con lo indicado por Bonachela (2001), quien señaló que mientras el bulbo húmedo estuvo en contacto con la radiación solar directa, la evaporación fue mayor, existiendo un consumo no demandado por las plantas, considerado hasta en un 43 \% para cultivos jóvenes.

\section{Conclusiones}

Al evaluar los sistemas de riego superficial y subsuperficial los resultados indican que el riego por goteo subsuperficial instalado a $20 \mathrm{~cm}$ de profundidad resulta en una importante reducción de uso del recurso hídrico, con efecto positivo en todas las variables evaluadas, además de ser el más eficiente en el uso de agua de riego.

\section{Referencias Bibliográficas}

Al-Ghobari, H. M. and Dewidar, A. Z. (2018). Integrating deficit irrigation into surface and subsurface drip irrigation as a strategy to save water in arid regions. Agricultural Water Management. 209, 55-61.

Ayars, J. E., Fulton, A. and Taylor, B. (2015). Subsurface drip irrigation in California-Here to stay? Agricultural Water Management. 157(31), 39-47.

Bonachela, S., Orgaz, F. O., Villalobos, F. J. and Fereres, E. (2001). Soil evaporation from drip-irrigated olive orchards. Irrig. Sci. 20(2), 65-71.

Bringas-Burgos, B., Mendoza-Muñoz, I., Navarro-González, C., González-Ángeles, A. y Jacobo-Galicia, G. (2020). Análisis de sistemas de riego por gravedad y goteo subsuperficial basada en una encuesta de muestra de conveniencia en el valle de Mexicali. VÍNCULOS-ESPE. 5(3), 13-32.

de Miguel, C. y Tavares, M. (2015). El desafío de la sostenibilidad ambiental en América Latina y el Caribe. Textos seleccionados 2012-2014. Cepal. ISBN: 978-92-1-057087-9 (publicación electrónica). 148 p.

Eranki, P. L., El-Shikha, D., Hunsaker, D. J., Bronson, K. F. and Landis, A. E. (2017). A comparative life cycle assessment of flood and drip irrigation for guayule rubber production using experimental field data. Industrial Crops and Products, 99(1), 97108.

FAO. (2019). Escasez de agua: Uno de los mayores retos de nuestro tiempo. Organización de las Naciones Unidas para la Alimentación y la Agricultura [en línea] disponible en: http://w.w.w.fao.org/sustainable-development-goals/goals-6/ es.www.fao.org/fao-stories/article/es /c/1185408. [consulta: 8 agosto 2020].

Ferreyra, R., Selles, G., Silva, H., Ahumada, R., Muñoz, I. y Muñoz, V. (2006). Efecto del agua aplicada en las relaciones hídricas y productividad de la vid 'Crimson Seedless'. Pesq. Agropec. Bras., 41(7), 1109-1118.

Girona, J., Mata, M., Del Campo, J., Arbonés, A., Bartra, E. and Marsal, J. (2006). The use of midday leaf water potential for scheduling deficit irrigation in vineyards. Irrigation Science, 24, 115-127.

Gomes de Andrade, J., Pacheco Seguí, J., Carlesso, R., Trois, C. y Knies, A.E. (2011). Perdidas de agua por evaporación en maíz con siembra convencional y directa para diferentes niveles de cobertura muerta. I. Resultados experimentales. Revista Ciencias Técnicas Agropecuarias, 20(2), 60-64.

Howell, T. A. (2001). Enhancing water use efficiency in irrigated agriculture. Agronomy Journal, 93, 281-289.

Hsiao, T. C. y Fereres, E. Maíz. pp. 117-126. En: Respuesta del rendimiento de los cultivos al agua. P. Steduto, T.C. Hsiao, E. Fereres y D. Raes (Eds.). Organización de las Naciones Unidas para la Alimentación y la Agricultura. Estudio FAO. (2012): Riego y Drenaje. $\mathrm{N}^{\circ} 66$.

INEC. (2019). Encuesta de Superficie y Producción Agropecuaria Continua (ESPAC) (2018). Instituto Nacional de Estadística y Censos [en línea] disponible en: https://www.ecuador encifras.gob.ec/documentos/web-inec/Estadisticas_agropecuarias/ espac/espac-2018/Presentacion \%20de\%20principales\%20resulta dos.pdf [consulta: 15 septiembre 2020].

INEC. (2020). Encuesta de Superficie y Producción Agropecuaria Continua (ESPAC) 2019. Instituto Nacional de Estadística y Censos [en línea] disponible en: https://www.ecuadorencifras. gob.ec/documentos/web-inec/Estadisticas_agropecuarias/ espac/espac-2019/Presentacion\%20de\% 20los\%20principales\%2 0resultados\%20ESPAC\%202019.pdf [consulta: 15 
septiembre 2020].

Lamm, F. R. y Trooien, T. P. (2003). Subsurface drip irrigation for corn production: A review of 10 years of research in Kansas. Irrigation Science, 22, 195-200.

Lucero-Vega, G., Troyo-Diéguez, E., Murillo-Amador, B., Nieto-Garibay, A., Ruíz-Espinoza, F. H., Beltrán-Morañes, F. A. y ZamoraSalgado, S. (2017). Diseño de un sistema de riego subterráneo para abatir la evaporación en suelo desnudo comparado con dos métodos convencionales. Agrociencia, 51, 487-505.

Montemayor, T. J. A., Gómez, M. A. O., Olague, R. J., Zermeño, G. A., Ruiz, C. E., Fortis, H. M., Salazar, S. E. y Aldaco, N. R. (2006). Efecto de tres profundidades de cinta de riego por goteo en la eficiencia de uso de agua y en el rendimiento de maíz forrajero. Те́c. Реси. Ме́x., 44, 359-364.

Montemayor, T. J. A., Olague, R. J., Fortis, H. M., Sam, B. R., Leos, R. J. A., Salazar, S. E., Castruita, L. J., Rodríguez, R. J. y Chavaría, G. J. A. (2017): Consumo de agua en maíz forrajero con riego subsuperficial, Torreón, Coahuila, México. Terra Latinoamericana, 25(2), 163-168.

Nieto C., C., Pazmiño Ch., E., Rosero, S. y Quishpe, B. (2018). Estudio del aprovechamiento de agua de riego disponible por unidad de producción agropecuaria, con base en el requerimiento hídrico de cultivos y el área regada, en dos localidades de la Sierra ecuatoriana. Siembra, 5(1), 051-070.

Papanatsiou, M., Petersen, J., Henderson, L., Wang, Y., Christie, J. M., Blatt, M. R. (2019). Optogenetic manipulation of stomatal kinetics improves carbon assimilation, water use, and growth. Science, 363(6434), 1456-1459.

Plan de Desarrollo y Ordenamiento Territorial de la Provincia de El Oro. PDOT 2014-2025. (2015). Gobierno Provincial Autónomo de El Oro-Ecuador.

Salomó, J., Sanmartín, J. M., Pérez, C., Maresma, A. y Lloveras, J. (2019). Riego por goteo subterráneo en cultivos de maíz y alfalfa. Estudio técnico-económico comparativo entre riego por inundación y riego por goteo enterrado. VIDA RURAL. Especial Maíz, 43-48.

Sánchez Delgado, M.Á. y Rivera Serna, L.G. (2018): Aplicación de dos modalidades de riego por goteo en el crecimiento y producción de camote (Ipomoea batatas L., variedad INIA 320). Anales Científicos, 79(1), 144-150.

Sinha, I., Buttar, G.S. and Brar, A.S. (2017). Drip irrigation and fertigation improve economics, water and energy productivity of spring sunflower (Helianthus annuus L.) in Indian Punjab. Agricultural Water Management, 185(1), 58-64.

Statistical Analysis System (SAS®) (2020). SAS STUDIO user's guide: Statistics. Version 15.1. SAS Institute Inc., Cary, NC. USA. Available: https://support.sas.com/documentation/onlinedoc /stat/ indexproc.html\#stat151

Steduto, P., Hsiao, T.C. and Fereres, E. (2007). On the conservative behaviour of biomass water productivity. Irrigation Science, 25, 189-207.

Thompson, T. L., Huan-cheng, P. and Yu-yi, L. I. (2009). The potential contribution of subsurface drip irrigation to watersaving agriculture in the western USA. Agric. Sci. China. Vol. 8, 850-854.

WWAP. (2018). Informe Mundial de las Naciones Unidas sobre el Desarrollo de los Recursos Hídricos (2018): Soluciones basadas en la naturaleza para la gestión del agua. United Nations World Water Assessment Programme. UNESCO (Paris). 168 p.

Zamora, S., Fenech, L., Ruiz, F., Pérez, W., López, A. (2007). Eficiencia en el uso del agua en maíz (Zea mays L.) con riego por goteo, en el Valle de la Paz, Baja California Sur, México. Revista Ciencias Técnicas Agropecuarias, 16(3), 33-36.

Zamora-Salgado, S., Ruiz-Espinoza, F. H., Beltrán-Morales, F. A., Fenech-Larios, L., Murillo-Amador, B., Loya-Ramírez, J. y Troyo-Diéguez, E. (2011). Régimen hídrico del maíz en una zona árida, determinado en porcentajes de evaporación. Tropical and Subtropical Agroecosystems, 13, 181-186. 


\section{REVISTA TECNICA}

DE LA FACULTAD DE INGENIERIA

UNIVERSIDAD DEL ZULIA

Vol. 44. №2, Mayo - Agosto, 2021

Esta revista fue editada en formato digital y publicada en Abril de 2021, por el Fondo Editorial Serbiluz, Universidad del Zulia. Maracaibo-Venezuela

www.luz.edu.ve

www.serbi.luz.edu.ve

www.produccioncientificaluz.org 